\title{
Histological and stereological characterization of brown trout (Salmo trutta) trunk kidney
}

\author{
A.D. Resende****, A. Lobo-da-Cunha******, F. Malhão**, F. Franquinho*, R.A.F. Monteiro****** \\ and E. Rocha****** \\ * North Polytechnic Health Institute (IPSN), Gandra, Portugal \\ ** Institute of Biomedical Sciences Abel Salazar (ICBAS), Porto University, Porto \\ *** Interdisciplinary Centre for Marine and Environmental Research (CIIMAR), Porto \\ albina.resende@ipsn.cespu.pt
}

The large macro- and microscopic variability in kidney morphology among fish makes it difficult to build a "universal" understanding on its function and structure [1]. Species-specific studies are thus needed. Thus, morphological study of brown trout trunk kidney was performed, considering potential seasonal and gender effects.

Three-year-old specimens of both genders were collected at four stages of their yearly reproductive cycle. Kidney pieces were processed for light and electron microscopy. With stereological methods, the relative volumes of renal components were estimated.

Qualitatively, the general nephron structure of brown trout was similar to that described for other glomerular teleost species, including other trouts. Quantitatively, however, differences in the relative volume of renal corpuscules, proximal tubule segments I and II (Fig. 1), collecting tubules, vacuolized tubules and new growing tubules (Fig. 2) were detected. In males from February, lower volumes of proximal tubules segments II, collecting tubules and renal corpuscules were found, paralleled by the highest values of vacuolized tubules. In those males, the greatest volumes of collecting tubules and renal corpuscules were found in September, whereas those of proximal tubules segment II existed in December. Females from September presented higher volumes of proximal tubule segments I, collecting tubules and renal corpuscules, comparing to the lowest values of February. In the latter, the highest values of new growing and vacuolized tubules existed. Differences between genders were detected in February, for the volume of the proximal tubule segments I, in May and December for the volume of proximal tubule segments II, and in December for the volume of renal corpuscules. Despite seasonal changes, only two linear correlations were found in females between the gonado-somatic index and some of the renal portions volumes, whereas more linear correlations between the latter and the reno-somatic index were found in both genders.

Thus, we verified that some brown trout renal components undergo gender dependent seasonal variations suggesting a morphological adaptation of the components to accomplish physiological needs [2]. These findings not only constitute a baseline for launching studies to know which factors govern the morphological variations and their functional consequences, but are also relevant for interpreting abnormal changes in toxicological contexts.

\section{References}

[1] M. Elger et al., Urinary Tract. Ostrander GK, editor. London: Academic Press. (2000) 385.

[2] R. Reimschuessel, ILAR Journal 42 (2001) 285.

This study was supported by Fundação para a Ciência e Tecnologia (FCT), Project POCTI/46968/BSE/2002 and PhD grant SFRH/BD/4609/2001. 

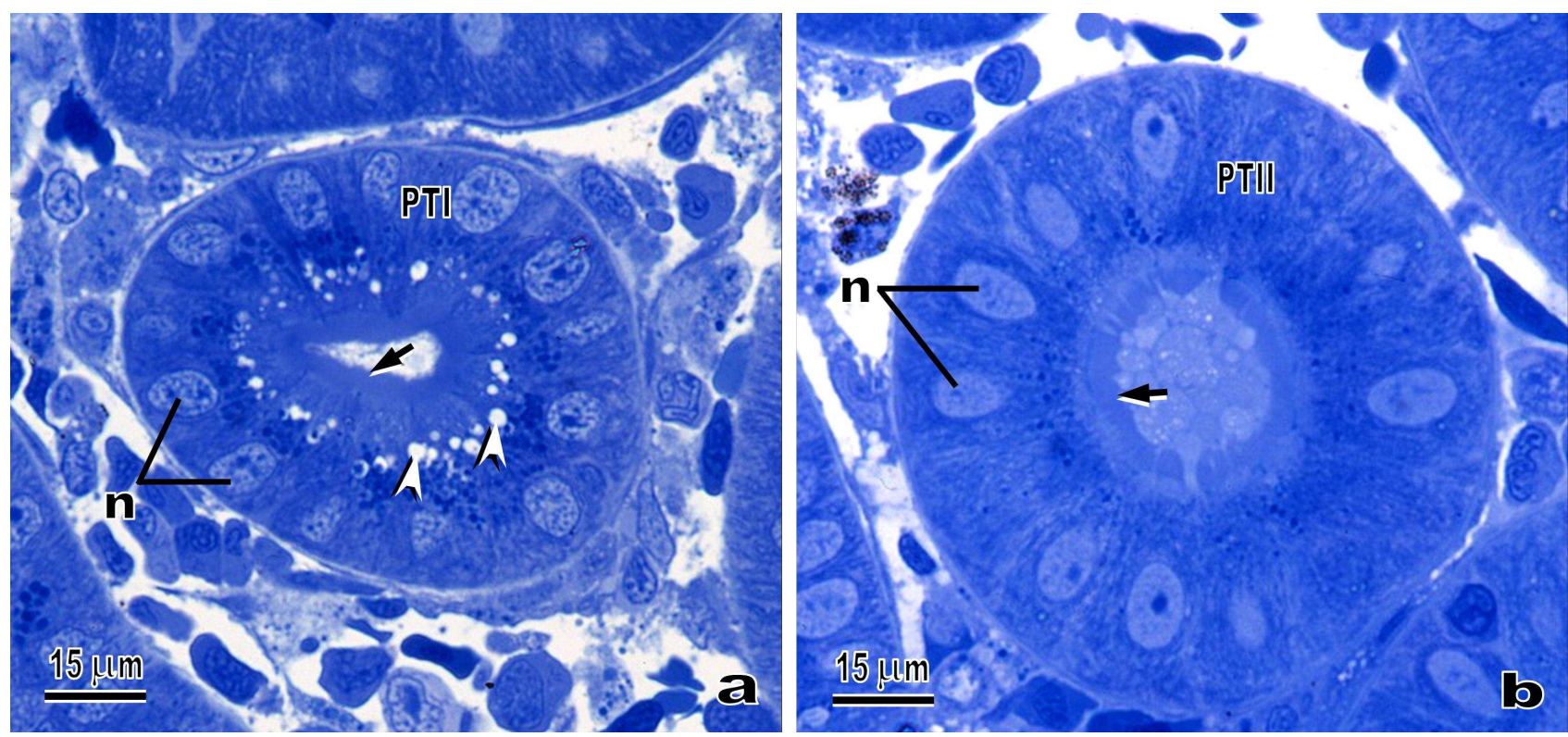

Fig. 1 Light micrographs of semithin sections from brown trout trunk kidney showing different nephron portions surrounded with haematopoietic tissue. a) Proximal tubule segment I (PT I). Observe the basally located nuclei (n) and the characteristic apical cell portion, plenty of endocytic vesicles (arrow heads) and an evident brush border (arrow). b) Proximal tubule segment II (PT II) showing the epithelial cells with central nuclei (n) and apical brush border (arrow).
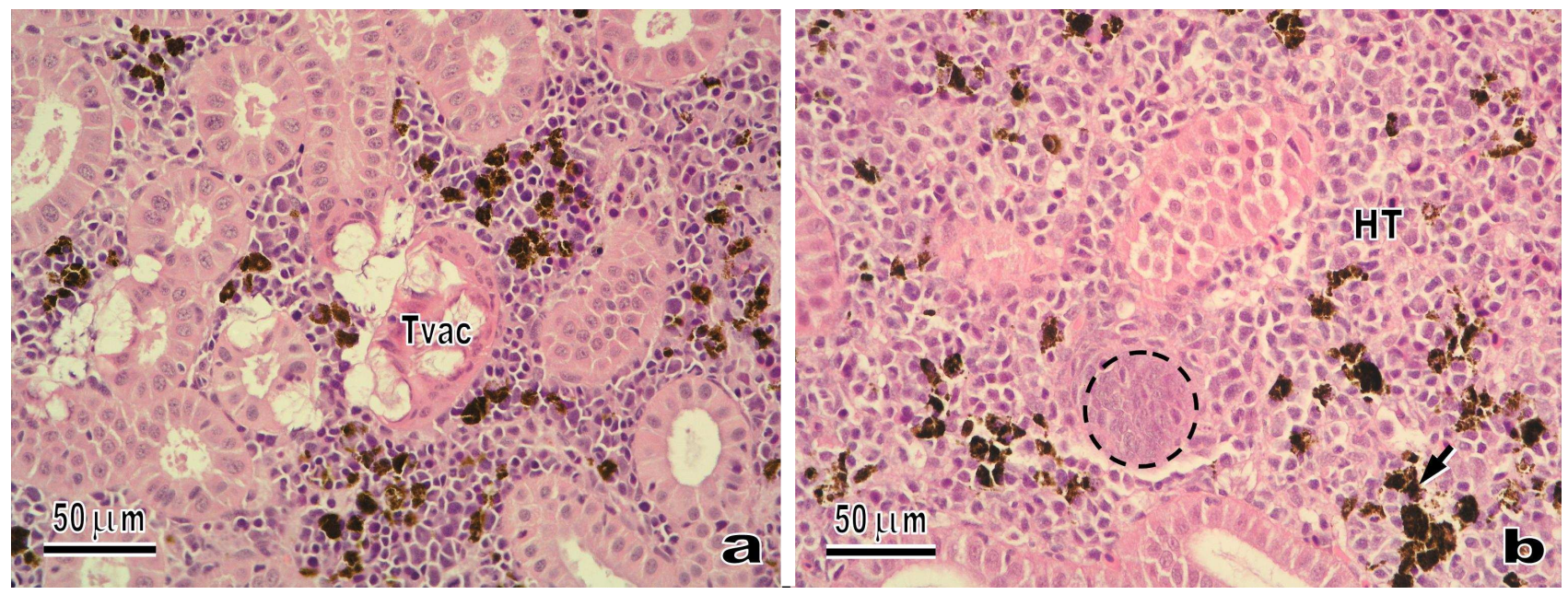

Fig. 2 Light micrographs of paraffin sections from brown trout trunk kidney, showing the different stereologically analysed components. a) Image with extremely rare vacuolized tubules (Tvac), with apparent peritubular vacuolization in some areas, surrounded with other renal tubule types and haematopoietic tissue. The vacuolized cells seem to be degenerating. Note that parasites were not seen associated with this peculiar condition. b) Photograph showing a tubule interpreted as in formation (circle) among other renal components, mainly haematopoietic tissue (HT) with some melanomacrophage aggregates (arrow). 